Isaac López-Laval, Alejandro Legaz-Arrese*, Keith George, Olga Serveto-Galindo, José María González-Rave, Joaquín Reverter-Masia and Diego Munguía-Izquierdo

\title{
Cardiac troponin I release after a basketball match in elite, amateur and junior players
}

DOI 10.1515/cclm-2015-0304

Received April 5, 2015; accepted June 8, 2015

\section{Abstract}

Background: Available scientific data related to cardiac troponin I (cTnI) release after intermittent exercise is limited. It is also of interest to determine what personal or environmental factors mediate the exercise-induced release of cTnI. This study had two objectives: 1) to examine the individual release of cTnI to a basketball match; and 2) to establish the influence of athlete status as well as biological age on cTnI release.

Methods: Thirty-six basketball players (12 adult elite [PBA]: 27.3 \pm 4.1 years, 12 adult amateur [ABA]: 29.6 \pm 2.9 years, and 12 junior elite [JBA]: $16.6 \pm 0.9$ years) participated in a simulated basketball match with serial assessment of cTnI at rest, immediately post- and at 1, 3, 6, 12, and $24 \mathrm{~h}$ post-exercise.

Results: The basketball match increased cTnI levels (pre: median [range]; 0.006 [0.001-0.026]; peak post: 0.024 [0.004-0.244] $\mu \mathrm{g} / \mathrm{L} ; \mathrm{p}=0.000)$, with substantial individual variability in peak values. PBA and JBA players showed higher baseline and post-exercise cTnI values than ABA (all $\mathrm{p}<0.05$ ). Peak cTnI exceeded the upper reference limit (URL) in the $26 \%$ of players (3 PBA; 6 JBA).

\footnotetext{
*Corresponding author: Alejandro Legaz-Arrese, Departamento de Fisiatría y Enfermería. C/Domingo Miral S/N. 50009. Zaragoza, Spain, Phone: +34 976761000, Fax: +34 976761720, E-mail: alegaz@unizar.es; and Section of Physical Education and Sports, University of Zaragoza, Zaragoza, Spain Isaac López-Laval: Section of Physical Education and Sports, University of Zaragoza, Zaragoza, Spain Keith George: Resarch Institute for Sport and Exercise Sciences, Liverpool John Moores University, Liverpool, UK Olga Serveto-Galindo: San Jorge Hospital, Huesca, Spain José María González-Rave: Section of Physical Education and Sports, University of Castilla La Mancha, Toledo, Spain Joaquín Reverter-Masia: Research Group Human Movement, University of Lleida, Lleida, Spain Diego Munguía-Izquierdo: Section of Physical Education and Sports, Faculty of Sport Science, Department of Sports and Computer Science, Pablo de Olavide University, Sevilla, Spain
}

Conclusions: The current results suggest that intermittent exercise can promote the appearance of cTnI and that this is potentially mediated by athlete status.

Keywords: athlete status; biological age; cTnI; intermittent exercise.

\section{Introduction}

The release of biomarkers of cardiamyocyte insult during and after exercise, such as cardiac troponins (cTn), is the topic of intense scientific enquiry and debate $[1,2]$. Evidence to date suggests a substantial number of athletes exceed the upper reference limit (URL) of cTn after long- [1, 3] and short-duration endurance exercise [4], as well as after moderate- [5] and low-intensity continuous exercise [6].

Beyond description, several recent studies have attempted to determine what personal and exerciserelated factors may promote an increase in cTn after exercise. An elevation in cardiac troponin I (cTnI) has been associated with increased exercise duration and intensity [5], exercise mode [7]; training level [8] and biological age [9]. In many of these areas data is contradictory.

For example, available scientific data related to cTn release after intermittent exercise, such as soccer [10, 11], rugby [12], basketball [13] or floorball [14] is limited, often poorly controlled [12] and with limited sampling times post-exercise [11, 12, 14]. Consequently this has produced contradictory data. Further it has been proposed that less experienced endurance athletes are more likely to exhibit detectable cTn levels than more experienced athletes after exercise [8,15-19]. These data are largely derived from field-base studies with limited post-exercise sample points in amateur runners that may underestimate peak cTn release [20]. The cTn release in professional elite athletes is less well understood with a few, older data sets also limited by sampling frequency [21, 22]. Unlike most of the previous results, we have recently observed in untrained subjects that a controlled 
endurance training intervention resulted in higher preand post-exercise values of cTn [23]. Nevertheless, the influence of training level on cTn release has not yet been evaluated in a controlled study with disparate groups in terms of training or athletic status. Evidence to date also suggests increased cTn appearance after exercise in adolescent athletes, possibly due to their immature cardiac muscle $[9,18,19,24,25]$, although only Tian et al. [9] directly compared adult and adolescent athletes in a controlled study. Currently, it is unknown if the cTn response to intermittent exercise is similar in adults and adolescents.

In an attempt to resolve these issues we examined, employing multiple sampling points during $24 \mathrm{~h}$ recovery from exertion, the influence of a simulated basketball match on the cTnI appearance in players with different status/training level (elite and amateur) as well as adult and adolescent basketball players. We hypothesise that cTnI appearance during recovery would be increased in adolescent and amateur players.

\section{Materials and methods}

\section{Subjects and design}

A total of 36 male basketball players (12 adult, elite, professional players from Spanish ACB League; [PBA], 12 adult, amateur players from a local league $[\mathrm{ABA}]$, and 12 elite junior players [Spanish Junior Top-Division; JBA]) were recruited and gave personal (and parental in JBA) written informed consent to participate in a simulated basketball match with serial assessment of cTnI during the first $24 \mathrm{~h}$ of recovery. The study was approved by the Research Ethics Committee of the Government of Aragón (Spain), and all players and their parents were informed of the purpose, nature, testing procedures, possible risks, and their right to terminate participation at will. The study complies with the principles laid down in the Declaration of Helsinki, adopted by the 18th World Medical Assembly, Helsinki, Finland, June 1964, and recently amended at the 59th World Medical Assembly, Seoul, Korea, October 2008.
The characteristics of the study population are shown in Table 1. PBA had higher training history and current training volume than both ABA and JBA (all $\mathrm{p}<0.05$ ). By design JBA were younger but there was no difference in age between PBA and ABA.

All subjects attended the laboratory on three occasions. At a preliminary testing session, 1 week before the main study, body height was measured to the nearest $0.1 \mathrm{~cm}$ (SECA 225, SECA, Hamburg, Germany) and body mass was determined to the nearest $0.05 \mathrm{~kg}$ (SECA 861, SECA). A questionnaire was completed to obtain personal data, training history, and history of cardiac symptoms (if any). A 12-lead ECG was performed and exclusion criteria were significant cardiac history or pathological electrocardiograph. All players then performed the $20 \mathrm{~m}$ shuttle run test [26] with the objective to estimate $\mathrm{VO}_{2 \max }$ and determine maximal heart rate (HR) using a Polar Team 2 system (1.4.1, Polar Electro Oy, Kempele, Finland).

Prior to the initial laboratory visit and the main train all players were instructed to avoid strenuous training for $48 \mathrm{~h}$ before the test and to maintain their normal diet, hydration, and sleep routine. Moreover, subjects ate their last meal at least $2 \mathrm{~h}$ before the test and were asked not to ingest any potentially ergogenic product (i.e. caffeine).

For the second laboratory visit every group was divided in two teams to perform the match, and the players played in their usual position. The matches followed the regulations established by the International Basketball Federation (FIBA). Every team made a change every 4 min of actual game time, so all players participated for the same amount of time (32 of the $40 \mathrm{~min}$ actual playing time). All players performed a similar warm-up and treated the match as a normal competitive game. Strong and standardized verbal encouragement was offered during the matches to achieve and intensity of play similar to competition. HR was recorded continuously during the basketball match using a Polar Team 2 system (1.4.1, Polar Electro Oy). The matches began at 11.30 am and took place in an indoor basketball field at a temperature of $20{ }^{\circ} \mathrm{C}$ and relative humidity of $60 \%$. Venous blood samples were taken before, immediately after ( $5 \mathrm{~min}$ ), and 1, 3, 6, $12 \mathrm{~h}$ after exercise to assess serum cTnI. A final laboratory visit occurred the following day to collect a $24 \mathrm{~h}$ post-exercise blood sample.

Blood samples were drawn from an antecubital vein and quickly centrifuged. Serum and plasma were drawn off and stored at $-80{ }^{\circ} \mathrm{C}$ for later analysis. cTnI was analyzed from samples of EDTA plasma with the Access AccuTnI assay (Beckman Coulter, Fullerton, CA, USA). The imprecision profile of 839 duplicate samples showed $10 \%$ and $20 \%$ coefficient of variation values of 0.014 and $0.008 \mu \mathrm{g} / \mathrm{L}$, respectively. The URL for cTnI, defined as the 99th percentile of healthy participants, was $0.04 \mu \mathrm{g} / \mathrm{L}$ [27].

Table 1: Characteristics of the basketball cohorts.

\begin{tabular}{|c|c|c|c|c|c|c|c|}
\hline & Age, years & Body mass, kg & Height, $\mathrm{cm}$ & $\mathrm{VO}_{2 \max }, \mathrm{mL} / \mathrm{kg} / \mathrm{min}$ & $\begin{array}{r}\text { Basketball } \\
\text { training } \\
\text { history, } \\
\text { years }\end{array}$ & $\begin{array}{r}\text { Basketball } \\
\text { training } \\
\text { frequency, } \\
\text { sessions/week }\end{array}$ & $\begin{array}{r}\text { Basketball } \\
\text { training } \\
\text { volume, } \\
\text { h/week }\end{array}$ \\
\hline Adult elite (PBA; $\mathrm{n}=12$ ) & $27.3 \pm 4.1^{c}$ & $98.3 \pm 12.9^{a, c}$ & $199 \pm 7^{\mathrm{a}, \mathrm{c}}$ & $58 \pm 3$ & $17 \pm 5^{\mathrm{a}, \mathrm{c}}$ & $6 \pm 0^{\mathrm{a}, \mathrm{c}}$ & $16 \pm 0^{a, c}$ \\
\hline Adult amateur ( $A B A ; n=12)$ & $29.6 \pm 2.9^{b}$ & $83.8 \pm 12.9$ & $184 \pm 6^{b}$ & $56 \pm 7$ & $13 \pm 3^{b}$ & $4 \pm 1$ & $8 \pm 4$ \\
\hline Junior elite (JBA; $n=11)$ & $16.6 \pm 0.9$ & $82.8 \pm 10.3$ & $192 \pm 8$ & $58 \pm 3$ & $8 \pm 4$ & $4 \pm 0$ & $8 \pm 0$ \\
\hline
\end{tabular}

Data are mean \pm SD. aSignificant difference between PBA and ABA basketball players. ${ }^{\mathrm{b}}$ Significant difference between ABA and JBA basketball players. 'Significant difference between PBA and JBA basketball players. 


\section{Statistical analysis}

Statistical analyses were performed using the IBM Statistical Package of Social Sciences (IBM SPSS Statistics, v. 20.0 for WINDOWS). Data are expressed as the mean \pm SD unless otherwise stated. Kolmogorov-Smirnov tests were used to analyze for normal distribution and consequently cTnI data were log-transformed prior to inferential statistical testing. To measure the impact of sampling time during recovery (pre, $5 \mathrm{~min}, 1,3,6,12$, and $24 \mathrm{~h}$ post-exercise) and athletes status (PBA vs. ABA vs. JBA) upon cTnI, a mixed model two-way ANOVA were performed with post-hoc Bonferroni tests employed when appropriate. The association between an increase in cTnI (the difference between baseline and peak post-exercise value) and other relevant variables (e.g. baseline cTnI, mean and max exercise HR during simulated basketball play) were assessed using bivariate Pearson's product moment correlation coefficients in the entire study cohort. The values were considered to be significant if $\mathrm{p}<0.05$.

\section{Results}

The $\mathrm{HR}_{\max }$ attained during the $20 \mathrm{~m}$ shuttle run was not different between groups. The mean $\mathrm{HR}$ and $\% \mathrm{HR}_{\max }$ during the match was lower in PBA compared to ABA and JBA (Table 2).

In the junior basketball match one player was injured and replaced. This subject was excluded from final data analysis. A significant main effect of sampling time was observed for $\mathrm{cTnI}$ with increases, compared to pre-exercise, at 1-, 3-, 6-, 12-, and 24-h post-exercise $(\mathrm{p}=0.000)$ (Figure 1). A significant main effect of group was noted with both baseline and recovery cTnI lower in $\mathrm{ABA}$ when compared to both PBA and JBA ( $p=0.001)$. In support of this there were differences between groups in the peak post-exercise values of CTnI (ABA: median [range]; 0.015 [0.004-0.039]; PBA: 0.027 [0.007-0.244]; JBA: 0.041 $[0.012-0.208] \mu \mathrm{g} / \mathrm{L} ; \mathrm{p}=0.011)$. There was no significant group by time interaction term $(\mathrm{p}=0.174)$.

Compared with the basal levels, increased posteffort cTnI values were observed in all individuals. Despite this, individual variability in peak cTnI was

Table 2: Heart rate during the basketball match.

\begin{tabular}{lrr}
\hline & $\begin{array}{r}\text { Mean HR, } \\
\text { beats } / \text { min \% }\end{array}$ & HR $_{\text {max }} \%$ \\
\hline PBA $(n=12)$ & $150 \pm 11^{a, b}$ & $79 \pm 4^{a, b}$ \\
ABA $(n=12)$ & $168 \pm 9$ & $87 \pm 3$ \\
JBA $(n=11)$ & $167 \pm 10$ & $84 \pm 4$ \\
\hline
\end{tabular}

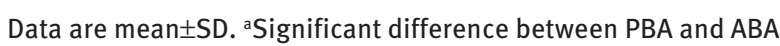
basketball players. ${ }^{b}$ Significant difference between PBA and JBA basketball players.
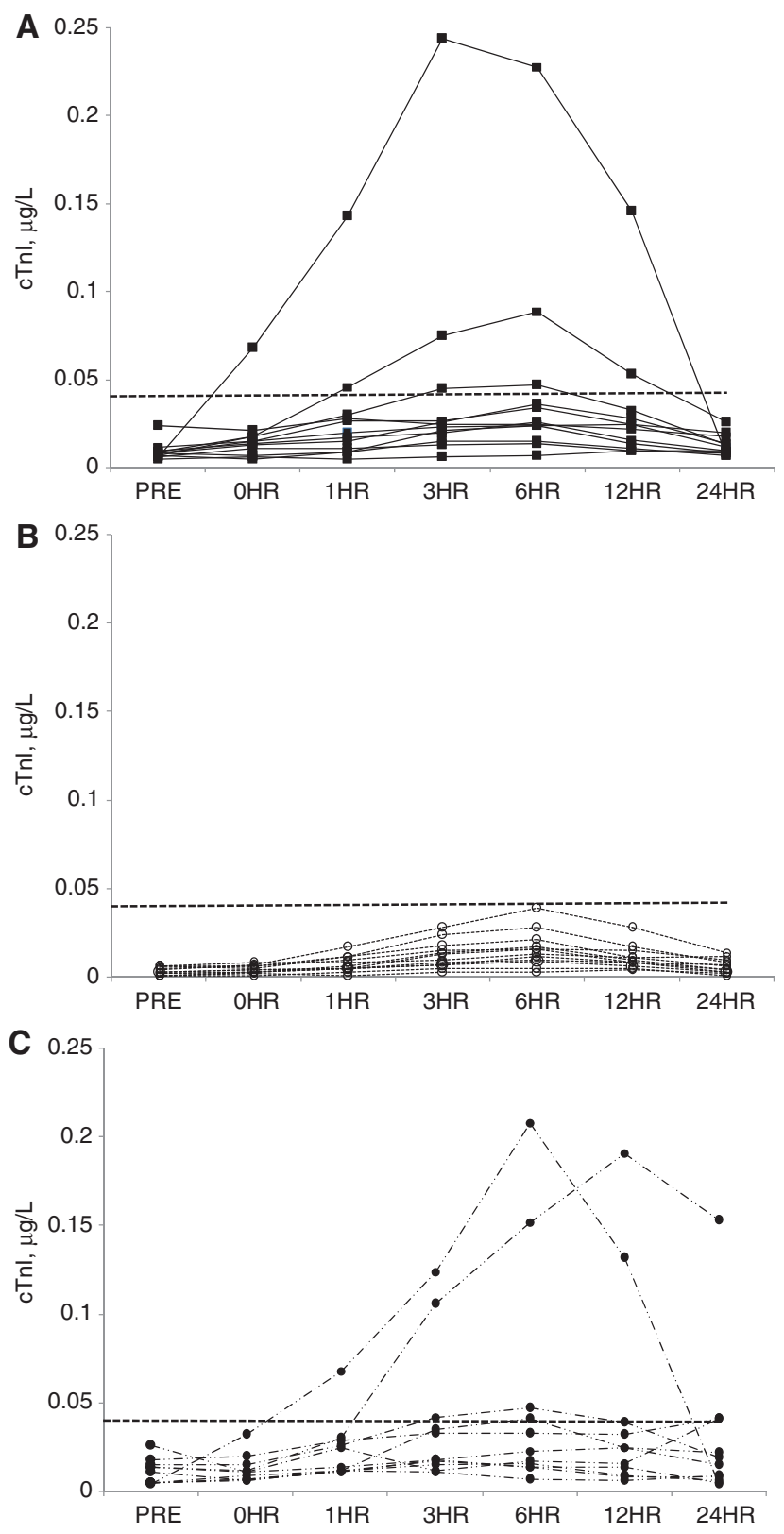

Figure 1: Individual data points for $\mathrm{CTnl}(\mu \mathrm{g} / \mathrm{L})$ in adult elite (PBA; $n=12)(A)$, adult amateur (ABA; $n=12)(B)$ and junior elite (JBA; $n=11)$ (C) basketball players at pre-exercise (PRE), as well as $5 \mathrm{~min}, 1,3,6$, 12 , and $24 \mathrm{~h}$ (0HR, $1 \mathrm{HR}, 3 \mathrm{HR}, 6 \mathrm{HR}, 12 \mathrm{HR}, 24 \mathrm{HR}$, respectively).

The horizontal dotted line is the upper reference limit (99th percentile) at $0.04 \mu \mathrm{g} / \mathrm{L}$.

noted with $26 \%$ of players (3 PBA and 6 JBA) exceeding the URL of cTnI (Figure 1). The maximum post-effort value was observed at $1 \mathrm{~h}$ in two individuals, $3 \mathrm{~h}$ in two individuals, $6 \mathrm{~h}$ in 23 individuals, $12 \mathrm{~h}$ in five individuals, and $24 \mathrm{~h}$ in two individuals suggesting a degree of heterogeneity in cTnI appearance "kinetics". It is also pertinent to note one JBA had an elevated cTnI at $24 \mathrm{~h}$ post-exercise. 
Peak post-exercise values of cTnI was not associated with player position, mean $\mathrm{HR}$ or $\% \mathrm{HR}_{\max }$, but was associated with baseline values $(\mathrm{r}=0.54, \mathrm{p}=0.001)$.

\section{Discussion}

This is the first study to investigate post-exercise cTnI "kinetics" after intermittent exercise performed in a controlled environment in distinct groups of athletes differentiated by athlete status and biological age. The key findings from our study are; 1) intermittent exercise resulted in a cTnI change in all athletes although the magnitude of response was heterogeneous; 2) the baseline and post-exercise cTnI was lowest in ABA compared to PBA and JBA; and 3) the cTnI response post-exercise was similar in elite adult and junior basketball players.

Previous studies documenting cTn release during intermittent exercise have reported contradictory results. A plausible explanation for this contradictory evidence has been the limited and varied number of biomarker sampling points employed in past studies. Whilst the current study observed an increased in cTnI post-exercise in all participants this runs contrary to both George et al. [12] and Rahnama et al. [11] who did not observe any cTnI increase. The likely explanation for this discrepancy was the use of a simple pre-post exercise sampling regime in previous work. The current data supports Nie et al. [13], in basketball players, and Carranza-García et al. [10], in futsal players, who reported an increase in cTn several hours after simulated match. Consequently, our findings show, in line with continuous endurance exercise studies [4, 20], the importance of obtained multiple blood samples during recovery to observe the maximum posteffort value.

Individual heterogeneity of peak cTnI was observed with $26 \%$ of the players presenting with one cTnI value over the URL. This percentage is lower than reported after endurance events, such as marathon [7] or cycling [3], and the more modest changes in the current study likely reflect the low total exercise duration and volume compared to many previous endurance and ultra-endurance activities [7]. Previous studies have noted that the increase in cTnI or cTnT is mainly mediated by the exercise duration when the intensity is controlled $[5,28]$.

Our results support heterogeneity in peak cTnI appearance and analysis of time to peak cTnI suggest some degree of variance in the pattern or "kinetics" of cTnI appearance, although it is noted that all participants, bar one JBA, demonstrated a return to baseline levels at the $24 \mathrm{~h}$ assessment point. The one JBA with an elevated (above URL) cTnI at $24 \mathrm{~h}$ post-exercise was signand symptom-free and unremarkable in personal or exercise details. Several studies have suggested a single blood sample 3-4 h post-exercise will reflect cTn peak post-exercise [18, 24, 29]. Our results are somewhat different with the most common time for cTnI peak at $6 \mathrm{~h}$ post-exercise. These data suggest that time when the peak post-exercise value may be dependent upon exercise mode, duration and/or intensity and further work is required.

The pattern of cTn appearance and clearance postexercise runs contrary to changes in cTn observed in acute coronary syndromes. This suggests that post-exercise cTn levels may be related to a physiological rather than a pathological response after the exercise stimulus. The hypothesis has been stated that endurance exercise causes an increase in membrane permeability due to the physiological stress placed on the cell, inducing a transient cytosolic leakage due to membrane damage, rather than cardiomyocyte necrosis [2].

Recently, some studies have suggested that cTn release after exercise may be more pronounced in less trained subjects [8, 15-19] as a consequence of a lower myocardial work efficiency. Thus, cTn release during exercise in those with lower training levels or athlete status could be one consequence of the adaptive process in myocardial cells, similarly to the process observed in skeletal muscle, which protects them against future bouts of strenuous exercise $[15,16]$. Our results did not support this theory with PBA and JBA demonstrating greater post-exercise values than ABA, even when PBA achieved a slightly lower mean HR and $\% \mathrm{HR}_{\max }$ during the simulated game. These data are consistent with our recent controlled endurance training intervention [23] and a field-based study with marathoners [30].

The higher post-exercise cTnI values in PBA and JBA than ABA were associated with differences in baseline cTnI. The association between baseline and postexercise cTnI values have been previously reported [5, 31, 32]. In healthy population little attention has been focused to the variability of baseline cTn values. Mingels et al. [17] obtained significantly higher hs-cTnT in males than in females. The authors speculated that these differences were as a consequence of the heart size of men being larger than women. The same explanation could be used to justify the higher baseline and post-exercise cTnI values our PBA players (adults), because the size of heart is greater in athletes with higher performance [33, 34], although it is not clear if heart size is different in JBA when compared to older ABA. Recent work from Saravia et al. [30] has suggested a link between higher levels 
of inflammation and increased cTn in faster marathon runners. Further research into the factors associated with the inter-subject variability in the baseline and exerciserelated values of $\mathrm{cTn}$ are required.

The lack of differences between PBA and JBA players may indicate that biological age does not mediate significant differences in the cTn response to intermittent exercise in elite athletes. Some caution should be expressed in this comment based on the small, but statistically significant, differences in $\mathrm{CV}$ work undertaken during exercise in PBA when compared to JBA as well as that if you compare JBA to ABA (still a biological age comparison with similar weekly training volumes) then a difference is noted with JBA exhibiting a larger response than older ABA. This comparison compares more favorably to past work assessing the impact of biological age on post-exercise cTn appearance $[18,19,24,25]$. The complex interaction here of biological age, training volume and elite athlete status is complex to "un-pick" and requires further work.

Clinicians should be aware that the release of cTnI is not exclusive to long-term strenuous efforts. cTnI values above the URL can be observed after intermittent exercise, such as basketball match, with heterogeneity noted in peak and kinetic data. From a clinical perspective, there is a limited rationale for full cardiovascular examination all athletes with positive cTn concentrations in the absence of other clinical signs and symptoms. When evaluating cTn in an emergency setting, detailed information regarding any recent exercise activities should be obtained, especially in the first $24 \mathrm{~h}$ post-exercise.

In addition to comments above we note the inherent limitation of cross-sectional studies in that the observed differences in the values of cTnI between PBA and ABA may have resulted from differences other personal factors (e.g. genetic differences) as well as exposure to different training volumes. To resolve this issue it would be interesting to observe the impact of a training program on exercise-induced cardiac biomarker release.

In this study, we highlighted that intermittent highintensity exercise, as basketball match, resulted in a cTnI change in all athletes although the magnitude of response was heterogeneous. In addition, our results did not support the hypothesis of previous studies suggesting that $\mathrm{cTnI}$ release after exercise may be more pronounced in less trained subjects [8]. We also observed that the pattern of cTnI appearance and clearance postexercise runs contrary to changes in cTn observed in acute coronary syndromes. This suggests that post-exercise cTn levels may be related to a physiological rather than a pathological response after the exercise stimulus. Finally, this investigation prompts further research into the factors associated with the inter-subject variability in the baseline and exercise-related values of cTnI.

\section{Conclusions}

Our results show that intermittent exercise, in this case a simulated basketball match, resulted in an increase in post-exercise cTnI in all athletes with a varied peak and time-to-peak cTnI. The second key finding was that athlete status mediated cTn appearance with elite athletes presenting with higher values than amateur athletes.

Acknowledgments: The authors would like to thank all the participants for their participation in this study.

Author contributions: All the authors have accepted responsibility for the entire content of this submitted manuscript and approved submission. ALA, KG and DMI conception and design of research. ILL, OSG, JMGR and JRM performed experiments. ILL and DMI analyzed data. DMI prepared figures. ILL drafted manuscript. ALA, KG and DMI edited and revised manuscript. All authors read and approved the final manuscript.

Financial support: This study was supported by the DEP 2010-16767 grants from the Spanish Plan for Research, Development and Innovation $(\mathrm{R}+\mathrm{D}+\mathrm{i})$ MICINN.

Employment or leadership: None declared.

Honorarium: None declared.

Competing interests: The funding organization(s) played no role in the study design; in the collection, analysis, and interpretation of data; in the writing of the report; or in the decision to submit the report for publication.

\section{References}

1. Scharhag J, George K, Shave R, Urhausen A, Kindermann W. Exercise-associated increases in cardiac biomarkers. Med Sci Sports Exer 2008;40:1408-15.

2. Shave R, Oxborough D. Exercise-induced cardiac injury: evidence from novel imaging techniques and highly sensitive cardiac troponin assays. Prog Cardiovasc Dis 2012;54:407-15.

3. Serrano-Ostáriz E, Legaz-Arrese A, Terreros-Blanco JL, López-Ramón M, Cremades-Arroyos D, Carranza-García LE, et al. Cardiac biomarkers and exercise duration and intensity during a cycle-touring event. Clin J Sport Med 2009;19:293-9.

4. Shave R, Ross P, Low D, George K, Gaze D. Cardiac troponin I is released following high-intensity short-duration exercise in healthy humans. Int J Cardiol 2010;145:337-9.

5. Serrano-Ostáriz E, Terreros-Blanco JL, Legaz-Arrese A, George K, Shave R, Bocos-Terraz P, et al. The impact of exercise duration and intensity on the release of cardiac biomarkers. Scand J Med Sci Sports 2011;21:244-9. 
6. Eijsvogels T, George K, Shave R, Gaze D, Levine BD, Hopman MT, et al. Effect of prolonged walking on cardiac troponin levels. Am J Cardiol 2010;105:267-72.

7. Shave R, George KP, Atkinson G, Hart E, Middleton N, Whyte G, et al. Exercise-induced cardiac troponin T release: a metaanalysis. Med Sci Sports Exer 2007;39:2099-106.

8. Neilan TG, Januzzi JL, Lee-Lewandrowski E, Ton-Nu TT, Yoerger DM, Jassal DS, et al. Myocardial injury and ventricular dysfunction related to training levels among nonelite participants in the Boston marathon. Circulation 2006;114:2325-33.

9. Tian Y, Nie J, Huang C, George KP. The kinetics of highly sensitive cardiac troponin T release after prolonged treadmill exercise in adolescent and adult athletes. J Appl Physiol 2012;113:418-25.

10. Carranza-García LE, George K, Serrano-Ostáriz E, Casado-Arroyo R, Caballero-Navarro AL, Legaz-Arrese A. Cardiac biomarker response to intermittent exercise bouts. Int J Sports Med 2011;32:327-31.

11. Rahnama N, Faramarzi M, Gaeini AA. Effects of intermittent exercise on cardiac troponin I and creatine kinase-MB. Int J Prev Med 2011;2:20-3.

12. George KP, Dawson E, Shave RE, Whyte G, Jones M, Hare E, et al. Left ventricular systolic function and diastolic filling after intermittent high intensity team sports. Br J Sports Med 2004;38:452-6.

13. Nie J, Tong TK, Shi Q, Lin H, Zhao J, Tian Y. Serum cardiac troponin response in adolescents playing basketball. Int J Sports Med 2008;29:449-52.

14. Wedin JO, Henriksson AE. Postgame elevation of cardiac markers among elite floorball players. Scand J Med Sci Sports 2014. [Epub ahead of print 2014 Aug 11]. doi:10.1111/sms.12304.

15. Fortescue EB, Shin AY, Greenes DS, Mannix RC, Agarwal S, Feldman BJ, et al. Cardiac troponin increases among runners in the Boston Marathon. Ann Emerg Med 2007;49:137-43.

16. Mehta R, Gaze D, Mohan S, Williams KL, Sprung V, George K, et al. Post-exercise cardiac troponin release is related to exercise training history. Int J Sports Med 2012;33:333-7.

17. Mingels A, Jacobs L, Michielsen E, Swaanenburg J, Wodzig W, van Dieijen-Visser M. Reference population and marathon runner sera assessed by highly sensitive cardiac troponin $T$ and commercial cardiac troponin T and I assays. Clin Chem 2009;55:101-8

18. Nie J, George KP, Tong TK, Gaze D, Tian Y, Lin H, et al. The influence of a half-marathon race upon cardiac troponin T release in adolescent runners. Curr Med Chem 2011;18:3452-6.

19. Tian Y, Nie J, Tong TK, Cao J, Gao Q, Man J, et al. Changes in serum cardiac troponins following a 21-km run in junior male runners. J Sports Med Phys Fitness 2006;46:481-8.

20. Middleton N, George K, Whyte G, Gaze D, Collinson P, Shave R. Cardiac troponin $T$ release is stimulated by endurance exercise in healthy humans. J Am Coll Cardiol 2008;52:1813-4.
21. Bonetti A, Tirelli F, Albertini R, Monica C, Monica M, Tredici G. Serum cardiac troponin T after repeated endurance exercise events. Int J Sports Med 1996;17:259-62.

22. König D, Schumacher YO, Heinrich L, Schmid A, Berg A, Dickhuth HH. Myocardial stress after competitive exercise in professional road cyclists. Med Sci Sports Exer 2003;35:1679-83.

23. Legaz-Arrese A, López-Laval I, George K, Puente-Lanzarote JJ, Mayolas-Pi C, Serrano-Ostáriz E, et al. Impact of an endurance training program on exercise-induced cardiac biomarker release. Am J Physiol Heart Circ Physiol 2015;308:H913-20.

24. Fu F, Nie J, George K, Tom TK, Lin H, Shi Q. Impact of 21-km run on cardiac biomarkers in adolescent runners. J Exerc Sci Fit 2010;8:61-6.

25. Nie J, Tong TK, George K, Fu FH, Lin H, Shi Q. Resting and post-exercise serum biomarkers of cardiac and skeletal muscle damage in adolescent runners. Scand J Med Sci Sports 2011;21:625-9.

26. Léger L, Gadoury C. Validity of the $20 \mathrm{~m}$ shuttle run test with 1 min stages to predict V02max in adults. Can J Sport Sci 1989;14:21-6.

27. Eggers KM, Lagerqvist B, Venge P, Wallentin L, Lindahl B. Persistent cardiac troponin I elevation in stabilized patients after an episode of acute coronary syndrome predicts long-term mortality. Circulation 2007;116:1907-14.

28. Fu F, Nie J, Tong TK. Serum cardiac troponin T in adolescent runners: effects of exercise intensity and duration. Int J Sports Med 2009;30:168-72.

29. Tian Y, Nie J, George KP, Huang C. Reproducibility of cardiac biomarkers response to prolonged treadmill exercise. Biomarkers 2014;19:114-20.

30. Saravia SG, Knebel F, Schroeckh S, Ziebig R, Lun A, Weimann A, et al. Cardiac troponin T release and inflammation demonstrated in marathon runners. Clin Lab 2010;56:51-8.

31. Klinkenberg LJ, Res PT, van Loon LJ, van Dieijen-Visser MP, Meex SJ. Strong link between basal and exercise-induced cardiac troponin T levels: do both reflect risk? Int J Cardiol 2012;158:129-31.

32. Legaz-Arrese A, George K, Carranza-García LE, MunguíaIzquierdo D, Moros-García T, Serrano-Ostáriz E. The impact of exercise intensity on the release of cardiac biomarkers in marathon runners. Eur J Appl Physiol 2011;111:2961-7.

33. Legaz-Arrese A, González-Carretero M, Lacambra-Blasco I. Adaptation of left ventricular morphology to long-term training in sprint- and endurance-trained elite runners. Eur J Appl Physiol 2006;96:740-6.

34. Legaz Arrese A, Serrano Ostáriz E, González Carretero M, Lacambra Blasco I. Echocardiography to measure fitness of elite runners. J Am Soc Echocardiog 2005;18:419-26. 